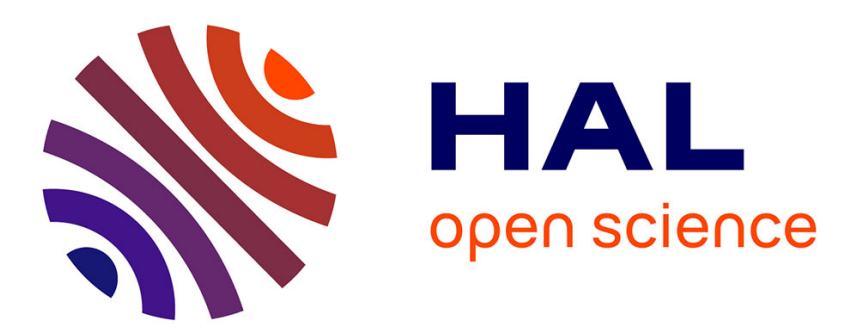

\title{
Matrix split-step resolution for propagation based on an exact spectral formulation
}

Rémi Douvenot, Christophe Morlaas, Alexandre Chabory, Bernard Souny

\section{To cite this version:}

Rémi Douvenot, Christophe Morlaas, Alexandre Chabory, Bernard Souny. Matrix split-step resolution for propagation based on an exact spectral formulation. APWC 2012, Topical Conference on Antennas and Propagation in Wireless Communications, Sep 2012, Cape Town, South Africa. pp 562-565, 10.1109/ICEAA.2012.6328690 . hal-01022310

\section{HAL Id: hal-01022310 \\ https://hal-enac.archives-ouvertes.fr/hal-01022310}

Submitted on 9 Sep 2014

HAL is a multi-disciplinary open access archive for the deposit and dissemination of scientific research documents, whether they are published or not. The documents may come from teaching and research institutions in France or abroad, or from public or private research centers.
L'archive ouverte pluridisciplinaire HAL, est destinée au dépôt et à la diffusion de documents scientifiques de niveau recherche, publiés ou non, émanant des établissements d'enseignement et de recherche français ou étrangers, des laboratoires publics ou privés. 


\title{
Matrix Split-Step Resolution for Propagation Based on an Exact Spectral Formulation
}

\author{
R. Douvenot, C. Morlaas, A. Chabory, and B. Souny*
}

\begin{abstract}
This paper introduces an alternative method to simulate wave propagation with a splitstep resolution. It is based on an exact formulation of the propagation operator. In this method, inspired by the classical parabolic equation formulation, the transforms from the spectral to the spatial representations are performed by a matrix multiplication. Although the matrix method is, in its current version, slower than usual split-step Fourier, it brings a new angle of view to perform the propagation simulation. Note that in the present version of the algorithm, the roughness is theoretically taken into account by modifying the Fresnel coefficient on the overall angular spectrum, and without spectral estimation or ray tracing to obtain the grazing angle.
\end{abstract}

\section{Introduction}

Large scale 2D electromagnetic simulations in the troposphere are classically carried out by a splitstep resolution of the parabolic wave equation (PWE), that derives from the parabolic approximation of Maxwell equations [1]. This propagation method takes into account the dielectric properties of the surface, the relief, and the atmospheric variations along the propagation path. It involves two main approximations: the backward propagation is neglected, and the power is supposed to be confined in a cone of revolution [2]. First introduced in the 40's by Leontovitch and Fock [3], it became popular when Dockery and Kuttler introduced the discrete mixed-term Fourier transform (DMFT) method to solve PWE [2]. One open problem is how the boundary condition at the ground level is considered, especially when the impedance condition depends on the angle of incidence. In the case of a rough surface for instance.

More recently, the theoretical background of PWE has been rewritten from square one [4]. A more rigorous formulation has been obtained in which the reflection coefficient is expressed in the spectral domain. Consequently, a rough ground modelled by Miller-Brown-Vegh formulation [5] is exactly described with this formulation.

The matrix formulation to model the propagation is based on the theoretical demonstration introduced by Chabory et al. [6]. This is close to Janaswamy's demonstration [4], but directly presents the formulation of the vertical operator.

\footnotetext{
${ }^{*}$ The authors are with ENAC/Telecom Lab/EMA, 7 av. E. Belin, 31055 Toulouse, France.
}

From this formulation, the matrix method is deduced. It is similar to the classical PWE, except that the successive transforms from the spectral to the spatial representations of the EM field are carried out through a linear system. A couple of simulations involving roughness are then performed for validation purpose.

\section{Matrix resolution}

\subsection{Exact formulation of the operator}

We suppose that the computation is carried out from 0 to $h$ in altitude. We also assume a rotational symmetry about the vertical axis. $\Gamma_{0}$ is the reflection coefficient of the smooth ground. We modify $\Gamma_{0}$ to obtain the reflection coefficient $\Gamma$ including roughness as [5]:

$$
\Gamma=\Gamma_{0} e^{-\zeta} I_{0}(\zeta)
$$

where $I_{0}$ is the modified Bessel function of order 0 , and

$$
\zeta \equiv \frac{1}{2}\left(\frac{4 \pi \delta_{\mathrm{r}} \sin (\theta)}{\lambda}\right)^{2},
$$

$\delta_{\mathrm{r}}$ being the height variation of the surface, $\theta$ the grazing angle, and $\lambda$ the wavelength.

Hereafter, $(r, z)$ are the cylindrical coordinates of the point where the field is computed. In [6], the exact spectral representation of the vertical operator for a domain of finite height is proved to be:

$$
\begin{aligned}
\Psi(r, z) & =\tilde{\Psi}^{\mathrm{p}}(r) e^{-j k_{z \mathrm{a}}^{\mathrm{p}} z}+ \\
& \sum_{n=1}^{+\infty} \tilde{\Psi}_{n}(r)\left(e^{j \frac{n \pi}{h} z}+\Gamma_{n} e^{-j \frac{n \pi}{h} z}\right)
\end{aligned}
$$

with the discrete spectral components:

$$
\begin{aligned}
& \tilde{\Psi}^{\mathrm{p}}(r)=\frac{2 j k_{z \mathrm{a}}^{\mathrm{p}}}{1-e^{-2 j k_{z \mathrm{a}}^{\mathrm{p}} h}} \frac{1}{1-\epsilon^{2}} \int_{0}^{h} \Psi(r, z) e^{-k_{z \mathrm{a}}^{\mathrm{p}} z} d z \\
& \tilde{\Psi}_{n}(r)=\int_{0}^{h} \frac{\Psi(r, z)}{2 h \Gamma_{n}}\left(e^{j \frac{n \pi}{h} z}+\Gamma_{n} e^{-j \frac{n \pi}{h} z}\right) d z
\end{aligned}
$$

in which $k_{z \mathrm{a}}^{\mathrm{p}}=\sqrt{k_{\mathrm{a}}^{2} \epsilon /(1+\epsilon)}, k_{\mathrm{a}}$ being the wave number above the ground, and $\epsilon \equiv \varepsilon_{\mathrm{ra}} / \varepsilon_{\mathrm{rg}}$ the permittivity contrast between air and ground. The first term of equation (3) corresponds to the pole 
of $\Gamma$. It is related to the ground wave. If $\Gamma$ has no pole, this term is null and there is no surface wave. The other terms correspond to vertical wave numbers $k_{z \mathrm{a}}=\frac{n \pi}{h}$. Thus, $\Gamma_{n}=\Gamma\left(\frac{n \pi}{h}\right)$.

We now define a function $\mathcal{M}$, which expression varies following the value of $k_{z}$ as:

$$
\begin{aligned}
& \mathcal{M}\left(z, k_{z}\right)=\sqrt{\frac{2 j k_{z}}{1-e^{-2 j k_{z} h}}} \frac{1}{\sqrt{1-\epsilon^{2}}} e^{-j k_{z} z} \\
& \text { for } k_{z}=k_{z \mathrm{a}}^{\mathrm{p}}, \\
& \mathcal{M}\left(z, k_{z}\right)=\sqrt{\frac{1}{2 h \Gamma\left(k_{z}\right)}\left(e^{j k_{z} z}+\Gamma\left(k_{z}\right) e^{-j k_{z} z}\right)} \\
& \text { for } k_{z}=\frac{n \pi}{h}, n \in \mathbb{N}^{*}
\end{aligned}
$$

Consequently, $\Psi$ can be rewritten substituting (5) into (3) as:

$$
\begin{aligned}
\Psi(r, z) & =\sum_{k_{z}} \int_{0}^{h} \Psi\left(r, z^{\prime}\right) \mathcal{M}\left(z^{\prime}, k_{z}\right) \mathcal{M}\left(z, k_{z}\right) d z^{\prime} \\
& =\sum_{k_{z}}\left[\mathcal{M}\left(z, k_{z}\right) \int_{0}^{h} \Psi\left(r, z^{\prime}\right) \mathcal{M}\left(z^{\prime}, k_{z}\right) d z^{\prime}\right] .
\end{aligned}
$$

\subsection{Discretisation of the operator}

We suppose that the signal is described by the first $N_{k}$ harmonics, and discretised on $N_{z}$ samples along the vertical. We now obtain an approximation of $\tilde{\Psi}^{\mathrm{p}}$ and $\tilde{\Psi}_{n}$ as:

$$
\begin{aligned}
& \tilde{\Psi}^{\mathrm{p}}(r)=\frac{2 j k_{z \mathrm{a}}^{\mathrm{p}}}{1-e^{-2 j k_{z \mathrm{a}}^{\mathrm{p}} h}} \frac{1}{1-\epsilon^{2}} \sum_{m=0}^{N_{z}-1} \Psi_{m} e^{-j k_{z \mathrm{a}}^{\mathrm{p}} \delta_{z} m} \delta_{z}, \\
& \tilde{\Psi}_{n}(r)=\sum_{m=0}^{N_{z}-1} \frac{\Psi_{m}}{2 h \Gamma_{n}}\left(e^{j \frac{m n \pi}{N}}+\Gamma_{n} e^{-j \frac{m n \pi}{N}}\right) \delta_{z},
\end{aligned}
$$

$\delta_{z}$ being the vertical step.

For the sake of simplicity, we take $N_{k}=N_{z}=N$. We define the matrix $\mathbf{M}$ as being the discretised function $\mathcal{M}$ on the $N \times N$ samples. $\mathbf{M}$ is the transformation matrix from the spatial space to the spectral space and it is supposed invertible. We call $\boldsymbol{\Psi}(r)$ the field at distance $r$ discretised on the $N$ vertical points and $\tilde{\boldsymbol{\Psi}}(r)$ the same field expressed in the spectral domain on the $N$ first spectral components. Then,

$$
\begin{aligned}
\mathbf{M}^{-1} \boldsymbol{\Psi}(r) & =\tilde{\boldsymbol{\Psi}}(r), \\
\mathbf{M} \tilde{\boldsymbol{\Psi}}(r) & =\boldsymbol{\Psi}(r) .
\end{aligned}
$$

\subsection{Split-Step Resolution}

Following (9), the field on any vertical can be expressed in the spectral domain through the resolution of a linear system with the field as the right-hand-side. This multiplication plays the same role as the Fourier transform in the classical PWE method. Thus, a split-step matrix (SSM) algorithm is deduced. Spectral and spatial steps are added to (8) and (9) to compute the field at distance $r+\Delta r$ from the one computed at $r$ :

$$
\boldsymbol{\Psi}(r+\Delta r)=\mathbf{P}_{r} \mathbf{M} \mathbf{P}_{k} \mathbf{M}^{-1} \boldsymbol{\Psi}(r)
$$

with

$$
\mathbf{P}_{r}[i]=\exp \left(-j k \Delta r \frac{m^{2}\left(r, z_{i}\right)-1}{2}\right),
$$

and

$$
\mathbf{P}_{k}[i]=\exp \left(-j \Delta r\left[k-k_{r}\left(r, z_{i}\right)\right]\right),
$$

for $i \in\{1, \cdots, N\}$. Besides, $m$ is the modified refractive index, $k$ is the wave number, and $k_{r}=$ $\sqrt{k^{2}-k_{z}^{2}}$, with a negative imaginary part imposed.

The analogy between (10) and classical PWE is obvious. Note that no hypothesis on paraxiality is made. It is intrinsically supposed when separating the spectral and the spatial contribution in the SSM formulation [2]. As previously shown in [4], there are some values of $\Gamma$ for which the algorithm diverges.

Next section presents simulation results to validate the algorithm.

\section{Simulations}

A first simulation is carried out with the following settings: the frequency is $5 \mathrm{GHz}$, the antenna, set at $30 \mathrm{~m}$, is excited in horizontal polarisation. The atmosphere is considered as homogeneous. The propagation factor is computed in a window of $60 \mathrm{~km}$ in distance and $200 \mathrm{~m}$ in height with computation steps of $200 \mathrm{~m}$ and $0.4 \mathrm{~m}$, respectively. The ground is considered as sea $\left(\varepsilon_{r}=80, \sigma=4\right)$.

Figure 1 displays the computed propagation factor in the case of smooth sea (Figure 1a) and sea waves of RMS height $0.51 \mathrm{~m}$, corresponding to a wind of $10 \mathrm{~m} / \mathrm{s}$ (Figure 1b). One observes the effect of roughness that decreases the intensity of the interference pattern, especially for higher incidence angles. This phenomenon is also visible on the cuts in Figure 3a. This effect is obtained without computing the grazing angle, as the spectral reflection coefficient is intrinsically taken into account in the simulation at each step (equation 7).

As being in homogeneous atmosphere, these results can be compared to the Miller-Brown-Vegh model [5] that gives the theoretical propagation factor with regard to the ground dielectric properties and the roughness. Vertical cuts of the propagation factors, with the configuration presented above, are 


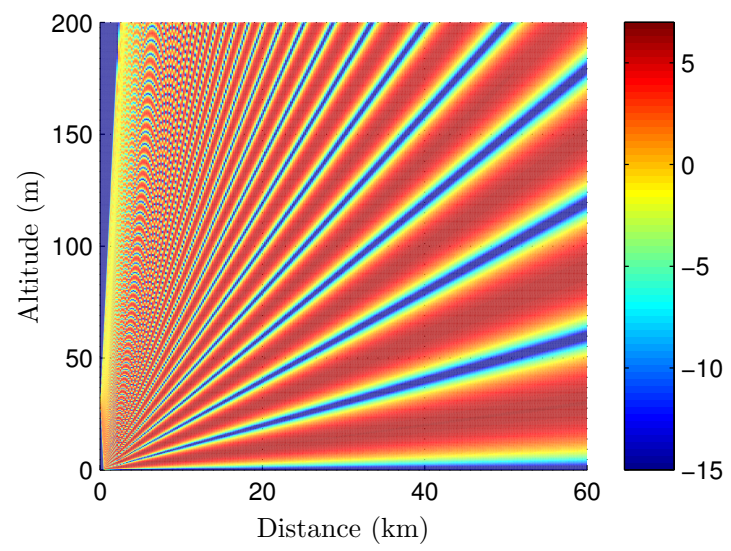

(a) smooth sea

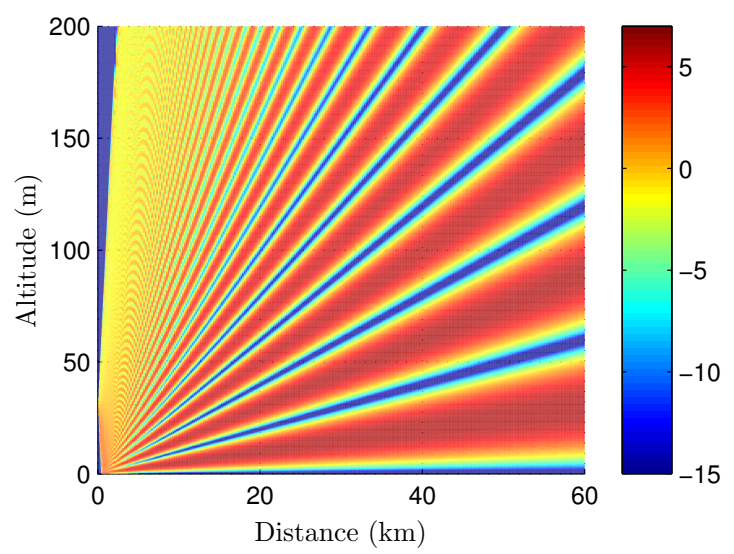

(b) rough sea

Figure 1: Propagation factor.

displayed in Figure 3 to compare the results obtained by SSM to the Miller-Brown-Vegh model.

For a better readability, in each Figure, the propagation factor obtained for wind speed $0 \mathrm{~m} / \mathrm{s}$ is shifted by $-10 \mathrm{~dB}$, the one obtained for wind speed $5 \mathrm{~m} / \mathrm{s}$ is not shifted, and the one obtained for wind speed $10 \mathrm{~m} / \mathrm{s}$ is shifted by $+10 \mathrm{~dB}$. Note that the wind speeds 0,5 , and $10 \mathrm{~m} / \mathrm{s}$ correspond to wave heights of $0 \mathrm{~m}, 0.13 \mathrm{~m}$, and $0.51 \mathrm{~m}$, respectively.

The propagation factors obtained with SSM are very close to the ones obtained with Miller-BrownVegh model. The roughness is correctly taken into account in the spectral domain, which shows the relevancy of the approach.

The second validation case is extracted from [4] (Figure 14). The frequency is $10 \mathrm{GHz}$, the antenna is set at $25 \mathrm{~m}$, in horizontal polarisation. The computation is carried out in a window of $150 \mathrm{~km}$ by $300 \mathrm{~m}$, with a horizontal step of $1 \mathrm{~km}$ and a vertical step of $0.2 \mathrm{~m}$. Dielectric properties are fixed to $\varepsilon_{r}=53, \sigma=17 \mathrm{~S} / \mathrm{m}$. The propagation factor is computed in presence of a surface-based duct, described by its modified refractivity:

$$
\begin{array}{rlr}
M(z) & =340.00-0.243 z & \text { for } z<50 \mathrm{~m} \\
& =321.95+0.118 z & \text { for } z>50 \mathrm{~m}
\end{array}
$$

The simulations are performed for two RMS ground heights: 0 and $1 \mathrm{~m}$.

Results are shown in Figure 2, similar to those obtained by Janaswamy. Small discrepancies remain, which is due to a coarser vertical sampling in the present simulation. Indeed, the matrix method requires more memory than classical SSF. Thus vertical sampling is fixed at 1500 points here. Despite this fact, results are similar and satisfying, considering the vertical sampling equal to $6.67 \lambda$.

\section{Conclusion}

In this paper, a new technique for PWE-like computation is presented. From the exact discretised formulation of the vertical operator, a matrix resolution is proposed. To perform the split-step, one goes back and forth from spectral to spatial domains through a linear system resolution. The reflection coefficient is exactly taken into account in the spectral domain. Note that hitherto, present method is less computationally efficient than classical Fourier transforms. However, significant improvements are expected.

\section{Acknowledgment}

The authors would like to thank Dr. Vincent Fabbro (ONERA) for discussions about PWE theory.

\section{References}

[1] M. Levy, Parabolic Equation Methods for Electromagnetic Wave Propagation, ser. IEE electromagnetic waves 45, IET, Ed., 2000.

[2] J. R. Kuttler and D. G. Dockery, "Theoretical description of the parabolic approximation/Fourier split-step method of representing electromagnetic propagation in the troposphere," Radio Sci., vol. 26, no. 2, pp. 381-393, 1991.

[3] M. A. Leontovitch and V. A. Fock, "Solution of propagation of electromagnetic waves along the Earth's surface by the method of parabolic equations," J. Phys. URSS, vol. 10, pp. 13-23, 1946.

[4] R. Janaswamy, "Radio wave propagation over a nonconstant immittance plane," Radio Sci., vol. 36, no. 3, pp. 387-405, 2001. 
[5] A. R. Miller, R. M. Brown, and E. Vegh, "New derivation for the rough-surface reflection coefficient and for the distribution of sea-wave elevations," in IEE Proc. H Microwaves, Optics and Antennas, vol. 131, no. 2, 1984, pp. 114-116.

[6] A. Chabory, C. Morlaas, R. Douvenot, and B. Souny, "An exact spectral representation of the wave equation for propagation over a terrain," in ICEAA, 2012.

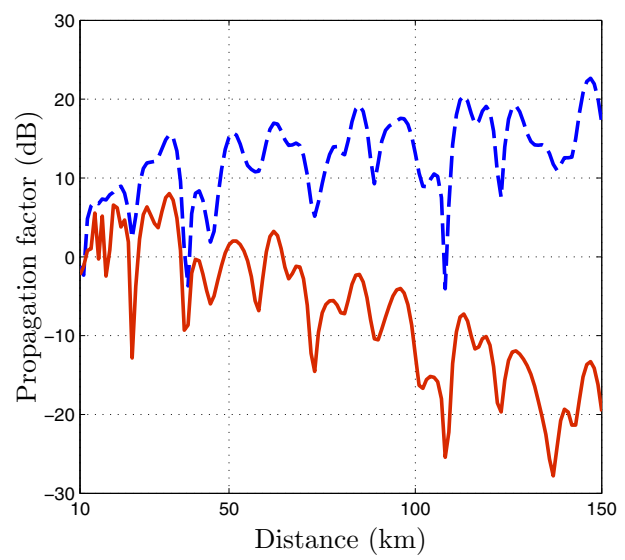

Figure 2: Propagation factor cuts for a receiver at $25 \mathrm{~m}$ along horizontal obtained by SSM. Dotted blue line is for smooth ground, continuous red line is for a RMS surface deviation of $1 \mathrm{~m}$.

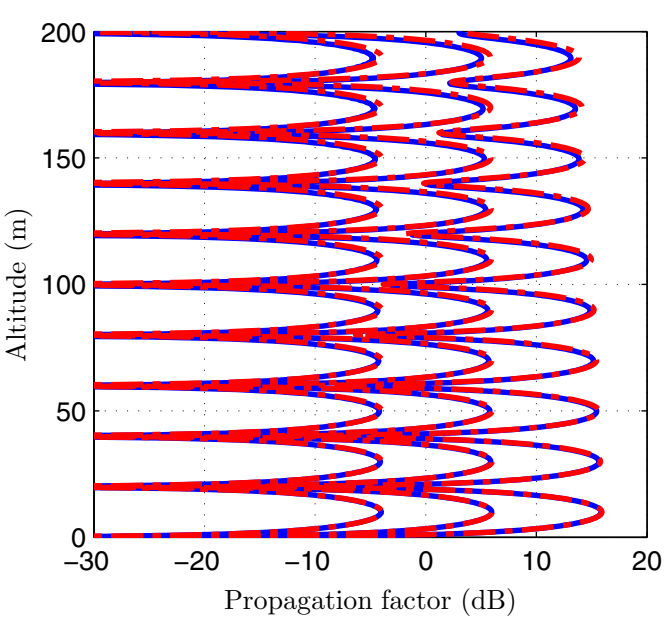

(a) Cuts at $20 \mathrm{~km}$

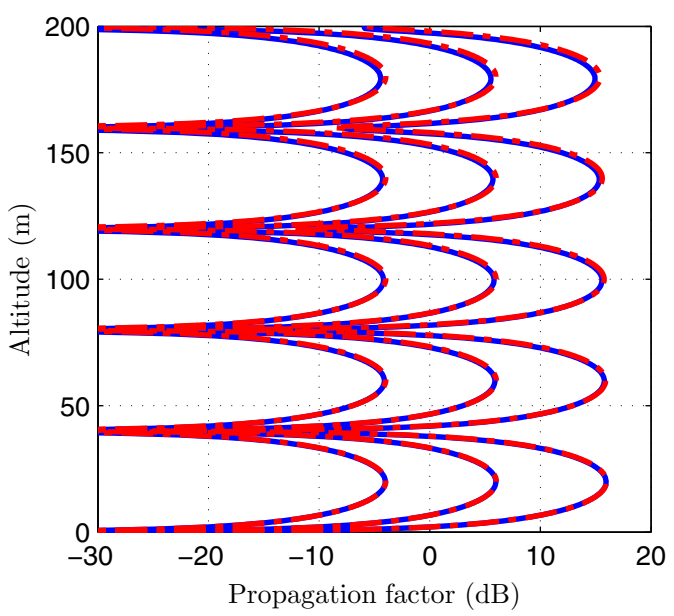

(b) Cuts at $40 \mathrm{~km}$

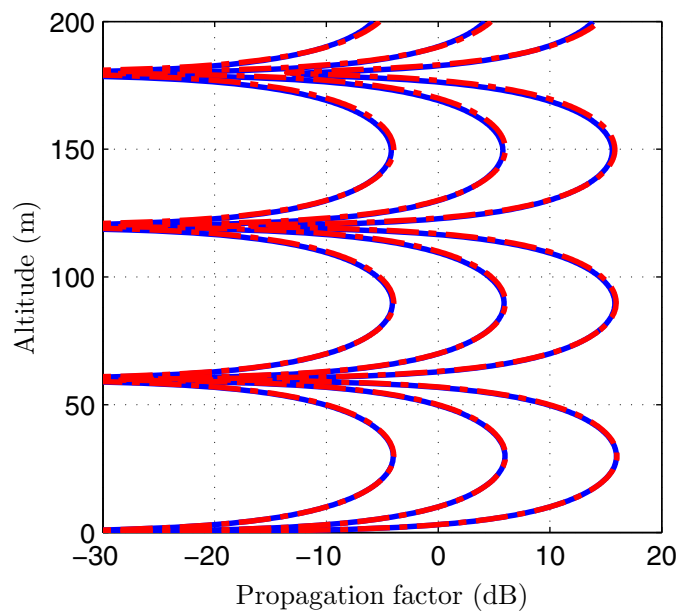

(c) Cuts at $60 \mathrm{~km}$

Figure 3: Propagation factor cuts. SSM results are the continuous blue line. Miller-Brown-Vegh results are the red dash-dot line. Results are plotted for wind speeds $0 \mathrm{~m} / \mathrm{s}(-10 \mathrm{~dB}), 5 \mathrm{~m} / \mathrm{s}$ (real value), 10 $\mathrm{m} / \mathrm{s}(+10 \mathrm{~dB})$. 